\title{
Seismic Wave Propagation Simulation in a Poro- elastic Medium Using Spectral Method Elements in MPI-GPU Cluster: Study Case of Anticline Reservoir Trap
}

\author{
Sudarmaji ${ }^{1}$, Indra Rudianto ${ }^{2}$, Muhammad Kautsar Rahmareza ${ }^{3}$ and Yosua \\ Alfontius ${ }^{4}$ \\ Department of Physics, Faculty of Mathematics and Natural Sciences, Gadjah Mada University, Yogyakarta, \\ Indonesia \\ 1ajisaroji@ugm.ac.id, 2indrarudianto.official@gmail.com, ${ }^{3}$ kautsar.rezha@gmail.com, \\ 4alfontiusyosua@gmail.com
}

\begin{abstract}
Modeling 2D seismic wave propagation using spectral element method on MPI-GPU clusters has been implemented and can complete the problem of the elastic poro-elastic medium model. Free surface boundary conditions and the absorption layer will use the CPML method. Seismic wave propagation simulation in the hybrid elastic poro-elastic medium has been carried out on hydrocarbon reservoir models with anticline type. In reservoir rocks that have a poro-elastic medium, there will be a fast and slow $\mathrm{P}$ wave. While in non-reservoir rocks that have elastic medium, only $\mathrm{P}$ fast waves are observed. The computation time for an anticline type reservoir model with dimensions of $1 \mathrm{~km} \times 1.5 \mathrm{~km}$ using MPI 4 nodes cluster with $48 \mathrm{CPU}$ cores is 3 minutes 48 second.
\end{abstract}

\section{INTRODUCTION}

In recent years, numerical simulation of wave propagation in fluid-saturated poroelastic media has received more attention as its importance in geophysical exploration and reservoir characterization. Biot's theory [1,2] is the basis for the numerical simulation of wave propagation in fluid saturated poroelastic media. The finite-difference (FD) method for Biot's equations has been formulated in several ways, central difference FD method in displacement [3], velocity-stress predictor corrector FD method [4]. An unsplit convolutional perfectly matched layer (CPML) is the stable new an absorbing boundary condition [5]. Numerical modeling of 2D seismic wave propagation in fluid-saturated porous media based on finite difference method using Graphics Processing Unit (GPU) has been implemented to simple hydrocarbon reservoir model [6].

Due to the irregular shape of reservoir distribution, implementation finite difference method has some limitation. Another numerical method which could address the irregular shape of reservoir distribution is a finite element and spectral element method. These methods have been successfully applied in many areas of seismic modeling [7-10]. Spectral element method also has been implemented to accommodate a considerable irregular shape of surface topography [11-12]. To accommodate the irregular shape of reservoir distribution, we use the spectral-element method (SEM) to simulate seismic wave propagation in the hydrocarbon reservoir model especially anticline reservoir model type. It uses a weak formulation for solving elastic wave equation and naturally incorporates the irregular shape of reservoir conditions. 


\section{THEORY}

\section{Strong Formulation}

Wave propagation in the poroelastic medium is defined by the following Biot equation:

$$
\begin{aligned}
& \bar{\rho} \partial_{t}^{2} \overline{\mathbf{u}}_{s}+\rho_{f} \partial_{t}^{2} \overline{\mathbf{w}}=\boldsymbol{\nabla} \cdot \overline{\mathbf{T}}+\mathbf{f}, \\
& m \partial_{t}^{2} \overline{\mathbf{w}}+\rho_{f} \partial_{t}^{2} \overline{\mathbf{u}}_{s}+\eta_{f} \mathbf{k}^{-1} \cdot \partial_{t} \overline{\mathbf{w}}=\boldsymbol{\nabla} \cdot \overline{\mathbf{T}}_{f}+\mathbf{f},
\end{aligned}
$$

with $\bar{\rho}$ is the average density defined by the equation

$$
\bar{\rho}=(1-\phi) \rho_{s}+\phi \rho_{f}
$$

with $\phi$ is porosity, $\rho_{s}$ is density of solid rock, and $\rho_{f}$ is the density of fluid. Solid rock displacement is symbolized by $\overline{\mathbf{u}}_{s}, \overline{\mathbf{w}}$ is the displacement of the fluid relative to the displacement of solid rock weighted by porosity,

$$
\overline{\mathbf{w}}=\phi\left(\overline{\mathbf{u}}_{f}-\overline{\mathbf{u}}_{s}\right)
$$

Average macroscopic stress $\overline{\mathbf{T}}$ defined by equation

$$
\overline{\mathbf{T}}=(1-\phi) \overline{\mathbf{T}}_{s}+\phi \overline{\mathbf{T}}_{f}
$$

The $m$ symbol in equation 2 is defined by $m=\rho_{f} c / \phi$, with $c$ is tortuisity, $\eta_{f}$ is fluid viskosity fluida, $k$ is permeability, $\overline{\mathbf{T}}_{f}$ is fluid stress, dan $\mathbf{f}$ is wave source. In isotropic media,

$$
\overline{\mathbf{T}}=\mathbf{G}: \boldsymbol{\nabla} \overline{\mathbf{u}}_{s}+C \boldsymbol{\nabla} \cdot \overline{\mathbf{w}} \mathbf{I}
$$

and

$$
\overline{\mathbf{T}}_{f}=-\bar{\rho}_{f} \mathbf{I}=\left(C \boldsymbol{\nabla} \cdot \overline{\mathbf{u}}_{s}+M \boldsymbol{\nabla} \cdot \overline{\mathbf{w}}\right) \mathbf{I},
$$

with

$$
\begin{aligned}
& C=\frac{\kappa_{s}\left(\kappa_{s}-\kappa_{f r}\right)}{D-\kappa_{f r}}, \\
& M=\frac{\kappa_{s}^{2}}{D-\kappa_{f r}}, \\
& D=\kappa_{s}\left[1+\phi\left(\frac{\kappa_{s}}{\kappa_{f}}-1\right)\right],
\end{aligned}
$$

with $\kappa_{s}$ is the bulk modulus of solid rock, $\kappa_{f}$ is the bulk modulus of fluid, and $\kappa_{f r}$ is the bulk modulus of frame. Tensor $\mathbf{G}$ defined by

$$
G_{i j k l}=\left(H-2 \mu_{f r}\right) \delta_{i j} \delta_{k l}+\mu_{f r}\left(\delta_{i k} \delta_{j l}+\delta_{i l} \delta_{j k}\right)
$$

with 


$$
H=\frac{\left(\kappa_{s}-\kappa_{f r}\right)^{2}}{D-\kappa_{f r}}+\kappa_{f r}+\frac{4}{3} \mu_{f r}
$$

with $\mu_{f r}$ is a shear modulus of the frame.

Wave source $\mathbf{f}$ could be written as tensor momen like :

$$
\mathbf{f}=-\mathbf{M} \cdot \boldsymbol{\nabla} \delta\left(\mathbf{x}-\mathbf{x}_{\mathbf{s}}\right) S(t)
$$

The location of the source point is denoted by $\mathbf{x}_{S}$, the distribution of the Dirac delta located in $\mathbf{x}_{S}$ is denoted by $\delta\left(\mathrm{x}-\mathrm{x} \_\mathrm{s}\right)$, and the time function of the source is denoted by $S(t)$.

\section{Weak Formulation}

In weak formulations, the equations of motion and implementation of boundary conditions are solved in an integral form. Weak formulations are obtained by multiplying dot equations 1 and 2 with any vector test $\widetilde{\mathbf{u}}$ and $\widetilde{\mathbf{w}}$ respectively, integrating partially on the volume model $\Omega$, and implementing the wave source in equation 4 . This gives

$$
\begin{aligned}
& \int_{\Omega} \bar{\rho} \widetilde{\mathbf{u}} \cdot \partial_{t}^{2} \overline{\mathbf{u}}_{\mathbf{s}} \mathrm{d}^{3} \mathbf{x}+\int_{\Omega} \rho_{f} \widetilde{\mathbf{u}} \cdot \partial_{t}^{2} \overline{\mathbf{w}} \mathrm{d}^{3} \mathbf{x} \\
&=-\int_{\Omega} \nabla \widetilde{\mathbf{u}}: \overline{\mathbf{T}} \mathrm{d}^{3} \mathbf{x}+\int_{\Gamma} \widehat{\mathbf{n}} \cdot \overline{\mathbf{T}} \cdot \widetilde{\mathbf{u}} \mathrm{d}^{2} \mathbf{x}+\mathbf{M}: \nabla \widetilde{\mathbf{u}}\left(\mathbf{x}_{\mathbf{s}}\right) S(t), \\
& \int_{\Omega} m \widetilde{\mathbf{w}} \cdot \partial_{t}^{2} \overline{\mathbf{w}} \mathrm{d}^{3} \mathbf{x}+\int_{\Omega} \rho_{f} \widetilde{\mathbf{w}} \cdot \partial_{t}^{2} \overline{\mathbf{u}}_{\mathrm{s}} \mathrm{d}^{3} \mathbf{x}+\int_{\Omega} \eta_{f} \widetilde{\mathbf{w}} \cdot\left(\mathbf{k}^{-1} \cdot \partial_{t} \overline{\mathbf{w}}\right) \mathrm{d}^{3} \mathbf{x} \\
&=-\int_{\Omega} \nabla \widetilde{\mathbf{w}}: \overline{\mathbf{T}}_{f} \mathrm{~d}^{3} \mathbf{x}+\int_{\Gamma} \widehat{\mathbf{n}} \cdot \overline{\mathbf{T}}_{f} \cdot \widetilde{\mathbf{w}} \mathrm{d}^{2} \mathbf{x}+\mathbf{M}: \nabla \widetilde{\mathbf{w}}\left(\mathbf{x}_{\mathbf{s}}\right) S(t) .
\end{aligned}
$$

with $\Gamma$ covering free surface boundaries, boundaries between acoustic-poroelastic, elastic-poroelastic, and poroelastic-poroelastic media layers.

\section{METHOD}

\section{D Model and Mesh Implementation}

The schematic model of anticline reservoir type and its mesh representation is shown in Figure 1. The dimension of the model is $1 \mathrm{~km} \times 1.5 \mathrm{~km}$ which contains some layers of rocks, such as: source rock (1), water reservoir (2), oil reservoir (3), gas reservoir (4), Seal Rock (5), Water Sand (6) and Shale (7). Zooming circle shows the interface between the elastic layer (seal rock) and poro-elastic layer (gas and oil reservoir). Each layer of rocks has their own physical properties. Table 1 shows physical properties of rock for the reservoir model. The mesh model has 12104 elements using interpolation for domains decomposition in the form of 4 th order polynomials. Spectral element sizes range from $2 \mathrm{~m}$ to $17 \mathrm{~m}$. Simulation is done by placing the source point at $(600,-10)$ and deploying 3 receivers at $\mathrm{x} 1(535,-10), \mathrm{x} 2(535,-450)$ and $\mathrm{x} 3(535,-750)$. 
a)

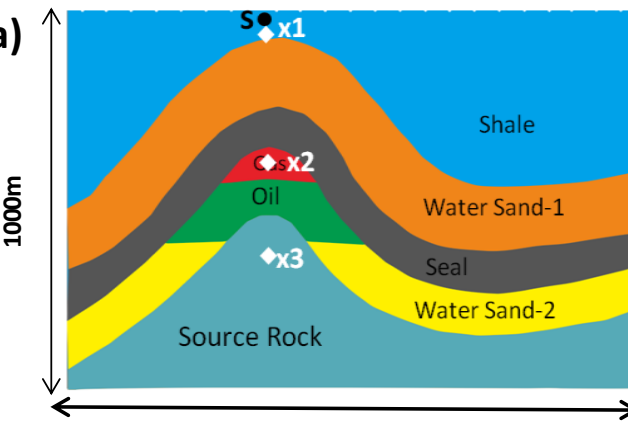

$1500 \mathrm{~m}$
Geophone

Source

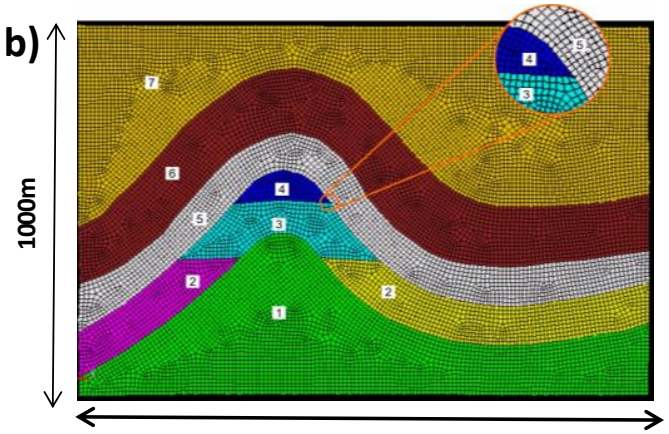

$1500 \mathrm{~m}$

Figure 1. a. Schematic of the anticline reservoir model. b. Mesh Implementation model.

Tabel 1. Physical properties of rock for the réservoir model

\begin{tabular}{|c|c|c|c|c|c|c|c|}
\hline \multirow{2}{*}{ Physical Properties } & \multicolumn{7}{|c|}{ Elastik } \\
\hline & \multicolumn{2}{|c|}{ Shale } & \multicolumn{2}{|c|}{ Water sand 1} & \multicolumn{2}{|l|}{ Seal } & Source \\
\hline $\operatorname{Vp}(\mathrm{m} / \mathrm{s})$ & & 3000 & & 3200 & & 3400 & 3600 \\
\hline $\operatorname{Vs}(\mathrm{m} / \mathrm{s})$ & & 1600 & & 1850 & & 1900 & 1950 \\
\hline$\rho s(\mathrm{~kg} / \mathrm{m} 3)$ & & 2750 & & 2900 & & 2950 & 2980 \\
\hline \multicolumn{8}{|c|}{ Poroelastik } \\
\hline Physical Properti & & gas & & oil & & wat & \\
\hline$\rho f(\mathrm{~kg} / \mathrm{m} 3)$ & & & 150 & & 650 & & 1000 \\
\hline$\mu$ & & & $33 \times 10^{\wedge} 9$ & & $6.2 \times 10^{\wedge} 9$ & & $6.57 \times 10^{\wedge} 9$ \\
\hline Kf & & & $3.4 \times 10^{\wedge} 7$ & & $7.41 \times 10^{\wedge} 8$ & & $2.24 \times 10^{\wedge} 9$ \\
\hline Ks & & 1.51 & $\times 10^{\wedge} 10$ & & $1.51 \times 10^{\wedge} 10$ & & $1.51 \times 10^{\wedge} 10$ \\
\hline Kfr & & & $96 \times 10^{\wedge} 9$ & & $9.86 \times 10^{\wedge} 9$ & & $1.28 \times 10^{\wedge} 10$ \\
\hline $\mathrm{a}$ & & & 1.2 & & 1.2 & & 1.2 \\
\hline$\phi(\%)$ & & & 0.2 & & 0.2 & & 0.2 \\
\hline${ }^{n}(\mathrm{~Pa} . \mathrm{S})$ & & & 0.00001 & & 0.03 & & 0.003 \\
\hline
\end{tabular}

\section{Simulation of the Anticline Reservoir Model}

We use the SPECFEM2D software package [6] and decompose the model into 12104 hexahedral elements. In each spectral element we use the polynomial degree of $N=4$, and thus each element contains $(N+1)^{3}=125$ Gauss-Lobatto-Legendre (GLL) integration points. Total number of GLL points in the model is $14,124,456$. The minimum and maximum distance between Gauss-LobattoLegendre integration points in the model is $2-17 \mathrm{~m}$ respectively. We use a Ricker wavelet as a source time function with dominant frequency is $20 \mathrm{~Hz}$. The time step used is $\Delta t=2.5 \times 10^{-5} \mathrm{~ms}$, and we propagate the signal for 50000 time steps, thus total simulation time is $1.25 \mathrm{~s}$.

The simulation is carried out on a GPU cluster with 4 NVIDIA QuadroK4000 graphics cards and 24 core processors Intel Xeon CPU E5-620 @ 2.4 GHz using MPI (Message Passing Interface) and CUDA (Compute Unified Device Architecture) at Computational and Seismic Laboratory, Geophysics Sub-Department, Gadjah Mada University. 


\section{RESULTS AND DISCUSSION}

Seismic wave propagation simulation is done by placing the source at the source point $(600 \mathrm{~m}, 10 \mathrm{~m})$ and the receiver is 60 units arranged in the lateral array form at a depth of $10 \mathrm{~m}$ from the surface to record reflection. The simulation uses a sampling time of $0.25 \times 10-5 \mathrm{~ms}$ with an iteration of 50000 times. The source wave is a Ricker wavelet with a dominant frequency of $20 \mathrm{~Hz}$. The free surface boundary conditions and absorption layer use the CPML method. Figure 2 shows a snapshot of the vertical component velocity propagation of the anticline type reservoir model at time $t=0.25 \mathrm{~s}$ to $\mathrm{t}=$ $0.5 \mathrm{~s}$.

a)

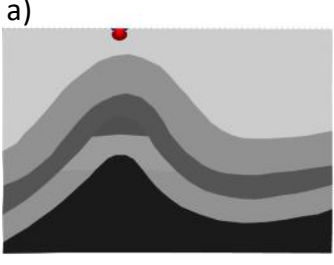

$t=0.25 \mathrm{~s}$

d)

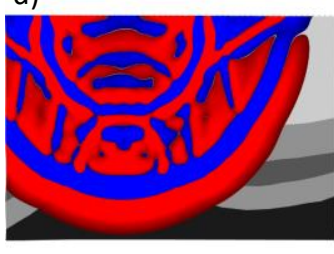

$t=0.3 \mathrm{~s}$ b)

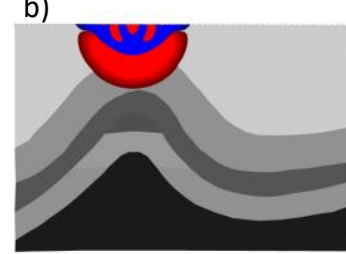

$t=0.1 \mathrm{~s}$
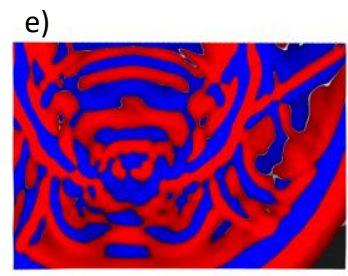

$t=0.4 \mathrm{~s}$

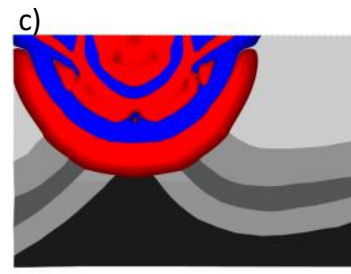

$t=0.2 \mathrm{~s}$

f)

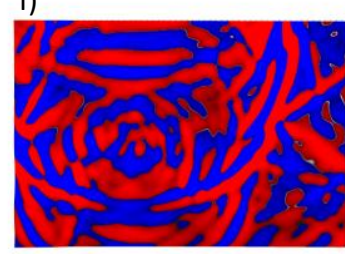

$t=0.5 \mathrm{~s}$

Figure 2. Snapshot of vertical component of velocity field propagation for the anticline reservoir model at time: a) $\mathrm{t}=0.25 \mathrm{~s}$, b) $\mathrm{t}=0.1 \mathrm{~s}, \mathrm{c}) \mathrm{t}=0.2 \mathrm{~s}, \mathrm{~d}$ ) $\mathrm{t}=0.3 \mathrm{~s}, \mathrm{e}) \mathrm{t}=0.4 \mathrm{~s}, \mathrm{f}) \mathrm{t}=0.5 \mathrm{~s}$

Figure 3 shows velocity seismogram at receiver X1, receiver X2 and receiver X3. Symbol $P_{d}^{f}$ is direct fast $\mathrm{P}$-wave, $P_{r 1}^{f}$ is $1^{\text {st }}$ reflection fast $\mathrm{P}$-wave between shale and water sand- $1, P_{r 2}^{f}$ is $2^{\text {nd }}$ reflection fast $\mathrm{P}$-wave between water sand- 1 and seal rock, $P_{r 3}^{f}$ is $3^{\text {rd }}$ reflection fast $\mathrm{P}$-wave between seal rock and gas reservoir, $P_{r 4}^{f}$ is $4^{\text {th }}$ reflection fast $\mathrm{P}$-wave between gas and oil reservoir, $P_{r 5}^{f}$ is $5^{\text {th }}$ reflection fast $\mathrm{P}$ wave oil reservoir and source rock, while $P_{d}^{S}$ is direct slow P-wave. Direct slow $\mathrm{P}$-wave $P_{d}^{S}$ is only observed in receiver $\mathrm{X} 2$ which is located in poroelastic medium as gas reservoir. Due to receiver X1 and X3 are deployed at elastic medium, so direct slow $\mathrm{P}$ wave are not observed. In the Biot's poroelasticity, it's well known there are two compressional waves; i.e.: fast $\mathrm{P}$ wave and slow $\mathrm{P}$ wave. Fast $\mathrm{P}$ wave is compressional wave from solid frame, while slow $\mathrm{P}$ wave is compressional wave from fluid filling the rock pore. 
a) Reciever X1

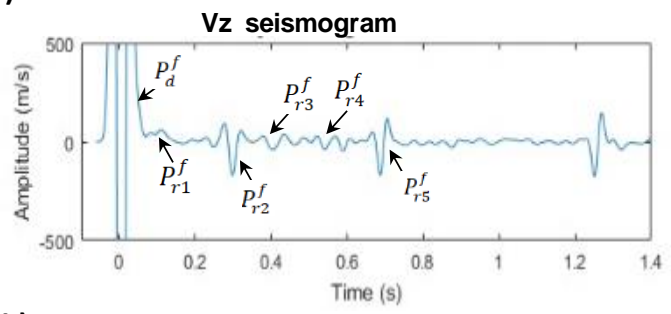

b) Reciever X2

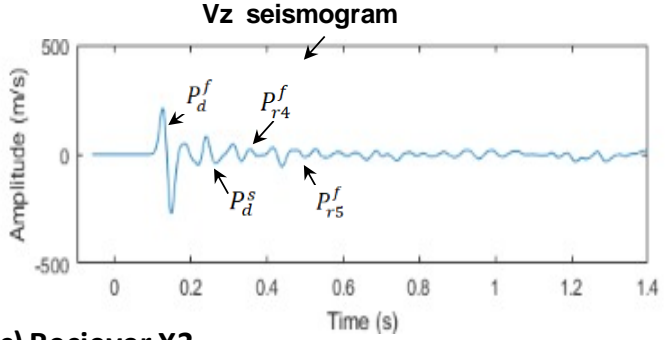

c) Reciever $X 3$

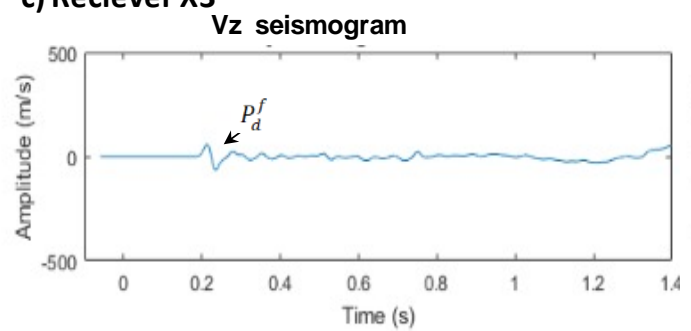

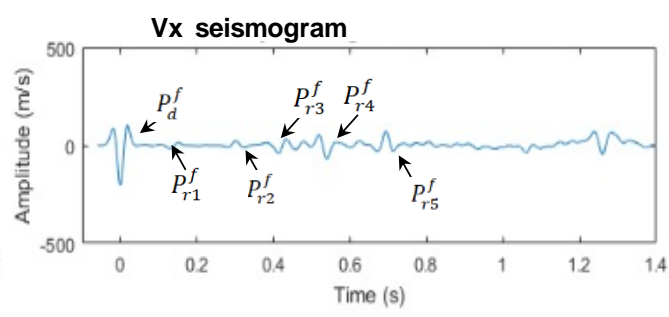
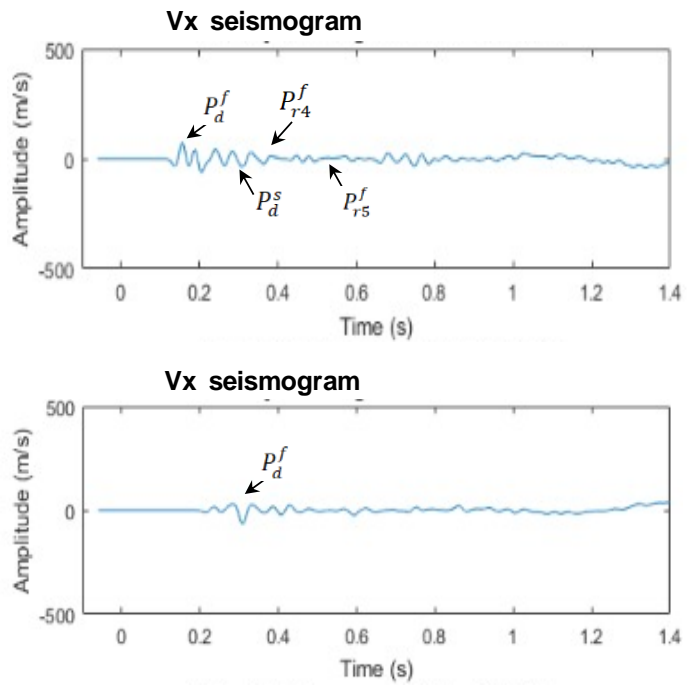

Figure 3. Velocity seismogram at: a) receiver $\mathrm{X} 1, \mathrm{~b})$ receiver $\mathrm{X} 2$ and c) receiver $\mathrm{X} 3$. Symbol $P_{d}^{f}$ is direct fast $\mathrm{P}$-wave, $P_{r 1}^{f}$ is $1^{\text {st }}$ reflection fast $\mathrm{P}$-wave, $P_{r 2}^{f}$ is $2^{\text {nd }}$ reflection fast $\mathrm{P}$-wave, $P_{r 3}^{f}$ is $3^{\text {rd }}$ reflection fast $\mathrm{P}$-wave, $P_{r 4}^{f}$ is $4^{\text {th }}$ reflection fast $\mathrm{P}$-wave, $P_{r 5}^{f}$ is $5^{\text {th }}$ reflection fast $\mathrm{P}$-wave and $P_{d}^{S}$ is direct slow P-wave

\section{CONCLUSIONS}

We presented numerical modeling 2D seismic wave propagation using spectral element method on MPI-GPU clusters has been implemented and can complete the problem of elastic-poro elastic medium model. Seismic wave propagation simulation in the hybrid elastic-poroelastic medium has been carried out on the hydrocarbon reservoir model with anticline type. In reservoir rocks that have a poro elastic medium, there will be a fast and slow $\mathrm{P}$ wave. While in non-reservoir rocks that have elastic medium, only $\mathrm{P}$ fast waves is the observed.

In our future works, we intend to develop more realistic mesh implementation, enlarge and make more detail the dimension of the model, and include all currently available relevant hydrocarbon reservoir information in our model.

\section{ACKNOWLEDGMENTS}

We would like to express our gratitude to PT. Chevron Indonesia for hardware donations given to Geophysics Study Program, Gadjah Mada University.

\section{REFERENCES}

1 Biot, M.A., 1956a, Journal of the Acoustical Society of America, v. 28, 168-178.

2 Biot, M.A., 1956b, Journal of the Acoustical Society of America, v. 28, 179-191.

3 Zhu, X., and McMechan, G.A., 1991, Geophysics, Vol. 56,No.3, p. 328-339. 
4 Dai, N.,Vafidis, A. and Kanasewich, E.R., 1995, Geophysics,Vol.60,No.2(March-April1995), p.327-340.

5 Martin, R, Komatitsch, D., and.,Ezziani, A. , 2008, Geophysics,vol. 73, no. 4, p. t51-t61

6 Sudarmaji, Sismanto, Waluyo and Soedijono B, 2016, AIP Conf. Proc.1755100001-1-7

7 Komatitsch D and Tromp J 1999 Geophys. J. Int.139 806-22

8 Komatitsch D, Erlebacher G, Göddeke D and Michéa D 2010J. Comp. Phys. 229 7692-7714

9 Komatitsch D, Liu Q, Tromp J, Süss P, Stidham C and Shaw J H 2004 Bull. Seismol. Soc. Am. 94 87206

10 Komatitsch D, Ritsema J and Tromp J 2002 Science 298 1737-42

11 Sudarmaji, Rudianto, I., Nurcahya, B.E, 2018, Journal of Physics: Conference Series 1011(1),012023

12 Sudarmaji, Rudianto, I., 2018, Journal of Physics: Conference Series 1011(1),012034 